\title{
Safeguarding NMA Enhanced Galileo OS Signals from Distance-Decreasing Attacks
}

\author{
Author Version
}

\author{
Kewei Zhang, KTH Royal Institute of Technology, Sweden \\ Panos Papadimitratos, KTH Royal Institute of Technology, RISE SICS, Sweden
}

\section{BIOGRAPHIES}

Kewei Zhang is a Ph.D. candidate with the Networked Systems Security (NSS) group at KTH Royal Institute of Technology, Stockholm, Sweden. He earned his MSc degree in Wireless Systems at KTH, Sweden. His research interests are GNSS security, wireless signal detection and estimation, and filtering.

Panos Papadimitratos is a professor with the School of Electrical Engineering and Computer Science (EECS) at KTH Royal Institute of Technology, Stockholm, Sweden, where he leads the Networked Systems Security (NSS) group. He earned his Ph.D. degree from Cornell University, Ithaca, New York, in 2005. His research agenda includes a gamut of security and privacy problems, with emphasis on wireless networks.

\begin{abstract}
Increased use of global satellite navigation systems (GNSS), for applications such as autonomous vehicles, intelligent transportation systems and drones, heightens security concerns. Civil GNSS signals are vulnerable to notably spoofing and replay attacks. To counter such attacks, cryptographic methods are developed: Navigation Message Authentication (NMA) is one such scheme, about to be deployed for Galileo E1 Open Service (OS); it allows receivers to verify the signal origin and protects navigation message integrity. However, NMA signals cannot fully thwart replay attacks, which do not require forging navigation messages. Classic replay attacks, e.g, meaconing, retransmit previously recorded signals without any modification, thus highly limiting the capacity of the adversary. Distance-decreasing (DD) attacks are a strong type of replay attack, allowing fine-grained individual pseudorange manipulation in real time. Moreover, DD attacks counterbalance processing and transmission delays induced by adversary, by virtue of shifting earlier in time the perceived (relayed) signal arrival; thus shortening the pseudorange measurements. In this paper, we first analyze how DD attacks can harm the Galileo E1 OSNMA service assuming the adversary has no prior information on the navigation message. Moreover,we propose a DD attack detection method based on a Goodness of Fit test on the prompt correlator outputs of the victim. The results show that the method can detect the DD attacks even when the receiver has locked to the DD signals.
\end{abstract}

\section{INTRODUCTION}

Civilian use GNSS signals are vulnerable to spoofing attacks, because of their public and predictable navigation messages. Countermeasures proposed for the infrastructure side contain signal authentication/encryption and navigation data authentication/encryption. In fact, commercial and military signals use signal encryption and/or navigation data encryption to restrict the access to the related signals, such as the GPS M-code signals, the Galileo Public Regulated Service (PRS) signals and the BeiDou authorized signals. But civilian signals have no such protection deployed yet. The European Global navigation Satellite systems Agency (GSA) has developed and begun testing the Galileo Open Service Navigation Message Authentication (OS NMA), which will enhance largely the receivers' ability against spoofing attacks. However, NMA cannot alone effectively protect receivers from replay attacks, including classic recording-and-replay attacks, security code estimation and replay attack (SCER) [1] and distance-decreasing (DD) attacks [2], [3]. Different from the classic recording-and-replay attacks, e.g., meaconing attack [4], DD attacks and SCER attacks are capable of changing pseudorange measurements for each individual satellite signal. Moreover, DD attacks can decrease the pseudorange measurements in real time, by a distance corresponding to a fraction of the bit/symbol length, thus compensating the processing delay introduced by the replaying devices.

Unlike the current unprotected GNSS signals, an attacker cannot spoof a receiver that uses NMA signals for positioning service because the attacker cannot generate a Message Authentication Code (MAC) [5], [6] without knowing the keys used by the satellites. The European GNSS Agency has been testing the E1-OSNMA service [7], [8], which is based on a combination of Time Efficient Stream Loss-tolerant Authentication (TESLA) [9] and public key cryptography. In such scheme, one messagebased secret value, i.e., MAC, is inserted into each message and the key used to generate this MAC is transmitted in the subsequent message. Thus, the receiver can validate the authenticity of the previous MAC based on the delayed disclosed key, which changes for each MAC. Therefore, one cannot craft its own signals to launch a spoofing attack since one cannot generate valid MAC. 
A meaconing attacker records signals corresponding to the frequency of the GNSS signal carrier wave and replays them to the victim later without modifying the signal contents. Consequently, all the signals arrive at the victim with same delay, which are weak points of the meaconing attack that the adversary cannot arbitrarily change the victim's position, velocity and timing (PVT). In contrast, the DD attacks can manipulate each individual signal separately and decrease each pseudorange measurement arbitrarily, with the adversary acting in two phases: early detection (ED) and late commit (LC). In the late commit phase, the attacker already transmits signals with a predefined data before having received actual data. When the actual data arrives, the attacker estimates the data value only based on the early fraction of the bit/symbol, and then switches to transmitting the estimated data. Consequently, the DD signal arrives at the victim receiver earlier than the actual signal. Therefore, a sophisticated attacker can leverage the DD attacks and classical replay attacks, to introduce both negative and positive delays to GNSS signals, to provide the victim with a false position or a false trajectory, without manipulating the cryptographically protected navigation messages.

The paper discusses how the adversary can possibly launch DD attacks on the Galileo E1OS-NMA services due to its special signal features and navigation message format. The results show that the adversary can use very short early detection time, based on the analysis of the symbol error rate in different noise environments, and then adjust the amplitude of the assembled signals with different approaches for the late commit phase for best adversarial results. The paper also analyzes the DD attacks to propose a countermeasure based on a statistical test on the prompt correlator outputs. Work based on monitoring the signal quality [10]-[13] propose test statistics based on checking the symmetric character of early correlator $E$ and late correlator $L$. The tests are a function of $E+L$ or $E-L$, with the null hypothesis that $E$ and $L$ are symmetrically located around the prompt correlator output in the absence of spoofing attacks. The authors have shown that the proposed hypothesis testing methods, e.g., sign test, goodness of fit test and ratio test metrics, can detect spoofing attacks during the periods that the spoofing signal correlator peaks are approaching the authentic peak and trying to take over the tracking lock. However, these tests are not able to detect the spoofing attacks when the receiver has already locked to the spoofing signals and shifted the correlator peaks away. In this paper, we have analyzed special mechanism of the DD attacks against the Galileo signals, with details explained in later sections. Hereafter, we propose a hypothesis testing method based on the analysis. When the victim has locked to the DD signals, the method can perfectly detect the DD attacks when the adversary wants to shorten a pseudorange measurement. With reasonable settings that the adversary transmits predefined signals $1.5 \mathrm{~ms}$ ahead before the reception of the authentic signals at the adversary, with early detection time being 500 chips, i.e., $0.5 \mathrm{~ms}$, and the processing delay being $1 \mathrm{~ms}$, the method detects the attacks for the whole simulation time.

The rest paper is organized as follows: we introduce the DD attacks in next section and shows the adversary model of the DD attacks on the GNSS signals. Hereafter, we provide a detailed analysis on early detection and late commit phases on the Galileo E1 OS signals, followed by the simulation results. Furthermore, we propose one DD attacks detection method based on a statistic test, which leads to conclusions of the paper.

\section{Adversary Model}

The distance-decreasing attacks are a type of physical layer attacks, as shown in Fig. 1a, to shorten the time-of-flight distance measurement between the honest signal transmitter (HTX) and the honest receiver (HRX), with the help of two components: early detection at the adversary receiver (ARX) and late commit at the adversary transmitter (ATX). The ATX starts transmitting signals based on a pre-defined bit/symbol value during $T_{L C}$ period. When the ARX receives the data, it estimates the data value only based on the early fraction of the symbol, $T_{E} D$. The ARX conveys the estimated value to the ATX. Upon receipt, the ATX switches the transmission to that value. Consequently, the DD signal arrives time $T_{D D}$ earlier than the authentic signal at the HRX, thus shortens the measured distance by $T_{D D} \cdot V_{C}$, between the HTX and the HRX. $V_{C}$ is the time speed and $T_{D D}=T_{L C}-T_{E D}-T_{d}$, with $T_{d}$ being the delay introduced by the adversary, including transmission and processing time.

In order to deploy DD attacks, the adversary needs to mount a pair of ED-LC on each attacked signal, as shown in Fig. 1b. The ARX is responsible for estimating the symbol value within $T_{E D}$ period and conveys the estimated symbol value together with other signal parameters to the ATX. The ATX is responsible for assembling new signals based on the ARX provided values, transmitting the assembled signals to the HRX. The communication between the ARX and the ATX is established with a fast link, which provides very small or even negligible delay, $T_{d}$.

The Galileo E1 OS signals, generated with Composite Binary Offset Carrier $\left(C B O C\left(6,1, \frac{1}{11}\right)\right)$ modulation, consist of two components: data component on channel $\mathrm{B}, e_{E 1 B}$, result of multiplying data with the spreading code, and one dataless component on channel $\mathrm{C}, e_{E 1 C}$, result of multiplying the primary code with the secondary code. The composite signal can be written as [14]:

$$
\begin{aligned}
S(t) & =\frac{1}{\sqrt{2}}\left\{e_{E 1 B}(t)\left(\alpha s c_{E 1-B O C(1,1)}(t)+\beta s c_{E 1-B O C(6,1)}(t)\right)-e_{E 1 C}(t)\left(\alpha s c_{E 1-B O C(1,1)}(t)-\beta s c_{E 1-B O C(6,1)}(t)\right)\right\} \\
& =\frac{1}{\sqrt{2}}\left\{S_{E 1 B}(t)+S_{E 1 C}(t)\right\}
\end{aligned}
$$




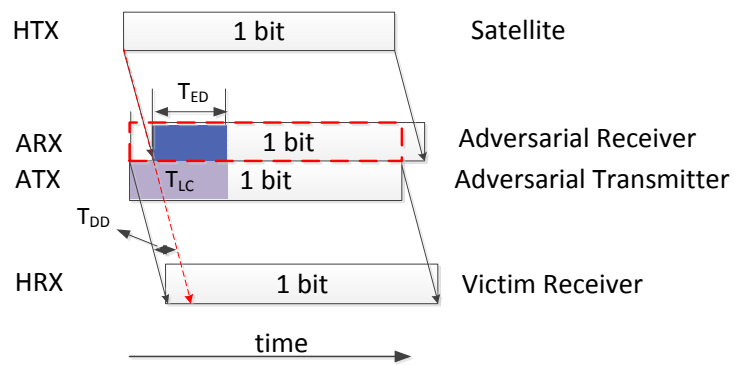

(a) Illustration of distance decreasing (DD) attack

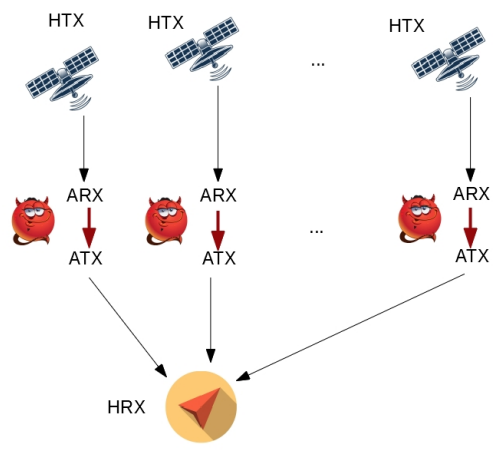

(b) Adversary illustration for DD attack on GNSS

Fig. 1: Distance-decreasing attacks on GNSS signals

where $s c_{X}(t)=\operatorname{sgn}\left(\sin \left(2 \pi R_{S, X} t\right)\right)$ is the sub-carrier with rate, $R_{S, X}, 1.023 \mathrm{MHz}$ for $s c_{E 1-B O C(1,1)}(t)$ and $6.138 \mathrm{MHz}$ for $s c_{E 1-B O C(6,1)}(t)$, and the composite signal is modulated on a carrier wave for transmission. When the ARX receives the signals, the baseband signals after the receiver front-end can be written as:

$$
R(t)=\sum_{k} \sqrt{P^{k}} S^{k}\left(t-\tau^{k}\right) \cos \left(2 \pi f_{d}^{k} t+\phi^{k}\right)+N(t)
$$

where $P$ is the power of the signal, $k$ is the satellite number index, $\tau$ is the time delay, $f_{d}$ is the Doppler frequency, $\phi$ is the carrier phase and $N(t)$ is the noise. The ARX estimates symbol values and signal parameters based on Eq. 2; the ARX has information of signal modulation and navigation message format, including preamble code, but not the message contents. The adversary can deploy the ARX at an open space area with good satellite visibility, in order to have good symbol estimation with high CNO and low interference. The ATX can be deployed at a position that is best for transmitting the assembled signals to the HRX; for instance, the ATX having good view of the HRX and/or close enough to the HRX in order to achieve similar reception power to that of authentic signals, allowing the ATX to adjust its transmission power accordingly.

Each ARX first needs to lock on one signal it wants to attack; the attacker has a precise estimation of parameters $\tau$, $f_{d}$, and $\phi$, with synchronization to the legitimate signal. These parameters are shared with the ATX, along with each symbol estimation. At the other side, each ATX broadcasts one signal, whose important parameters are the Doppler frequency and the bit/symbol value. The carrier phase is not important when the attacker has no intention to mount a phase alignment attack [15], but rather jams the HRX. One sophisticated adversary can implement a smooth takeover attack [16] without mounting a jamming attack in prior. However, a smooth takeover attack is hardly possible in practice because the attacker needs to know very precise information of carrier phase and code phase at the victim receiver location. Finally, the signals at the HRX can be written as:

$$
\begin{aligned}
R_{H R X}(t)= & \sum_{k} \sqrt{P^{k}} S\left(t-\tau^{k}\right) \cos \left(2 \pi\left(f+f_{d}^{k}\right) t+\phi^{k}\right)+ \\
& \sum_{k} \sqrt{P_{D D}^{k}} S\left(t-\tau_{D D}^{k}\right) \cos \left(2 \pi\left(f+f_{d, D D}^{k}\right) t+\phi_{D D}^{k}\right)+N(t)
\end{aligned}
$$

where the terms with DD subscript are for the assembled DD signals, otherwise for the authentic signals. $\tau_{D D}=\tau-T_{D D}$ indicates that the DD signals arrive $T_{D D}$ earlier than the authentic signals, and $P_{D D}$ is larger than $P$ in order to overshadow the authentic signals.

\section{Early Detection and late Commit Phases}

We first discuss the data component in $R(t)$, i.e., channel $\mathrm{B}$, for the data estimation. For one satellite signal, the digitalized data component, with sampling frequency $f_{s}$, is written as:

$$
r_{B}(n)=\sqrt{P} S_{E 1 B}(n-\tau) \cos \left(2 \pi f_{d} / f_{s} n+\phi\right)+N(n)
$$

This is used for the early detection at the ARX. 


\section{Early Detection}

The ARX, implementing the early detection phase, has already locked on to the available satellite signals. The adversary decides to start the DD attack at time $t_{0}$ : the ATX starts transmitting the DD signals at $t_{0}, T_{D D}$ earlier than the actual data reception at the ARX. $t_{0}$ should be aligned to the beginning of one symbol and one spreading code sequence, so that it is easier for the ATX to assemble the DD signals.

At time $t_{0}$, the ATX has very precise estimation of the Doppler frequency, code phase and carrier phase of last symbol, conveyed by the ARX. These parameters are used to assemble the signal for the current data, which is not known yet. Meanwhile, the ARX aims at determining the symbol value only over period $T_{E D}$, a fraction of the symbol duration. This is also called partial period correlation (PPC) [17], [18], in which one data symbol length is shorter than one spreading code sequence length. Naturally, the early detection phase degrades the ARX performance due to low SNR, not only due to the short integration period, but also the interference of other spreading codes due to the short correlation period. In this subsection, we first analyze the effects caused by the short integration period, then examine the interference among different spreading codes due to PPC.

The ARX can estimate the symbol value by taking the prompt correlator output as input: energy accumulation over period $T_{E D}$ after wiping off the carrier wave and the spreading code. The observation is a normalized result of baseband signal multiplying with the locally generated carrier and spreading code over the $T_{E D}$ period:

$$
x_{p}=\frac{1}{T_{E D} f_{s}} \sum_{k=p_{1}}^{p_{1}+T_{E D} f_{s}} r_{B}(k) S_{E 1 B}(k-\hat{\tau}) \cos \left(2 \pi \hat{f}_{d} / f_{s} k+\hat{\phi}\right)
$$

where $p_{1}$ is the first sample index of symbol $p ;\left\{\hat{\tau}, \hat{f}_{d}, \hat{\phi}\right\}$ are the estimated values obtained from the tracking outputs of previous symbol. As the receiver has very precise parameter estimation, it is coherent correlation over integration time $T_{E D}$ in Eq. 5, which is also a matched filter [19]. Due to its linearity, $x_{p}$, used to estimate the value of symbol $p$, can be written as:

$$
x_{p}=E b_{p}+n
$$

where $E$, energy, is a function of $T_{E D}, b_{p}$ is the value of symbol $p$, and $n$ is Gaussian noise with $n \sim N\left(0, \sigma_{x}^{2}\right)$. Three well-known estimation schemes are considered: maximum likelihood (ML), maximum a posterior (MAP) and minimum mean square error (MMSE).

The results based on different estimation schemes are derived in Appendices A and B. Having a prior information about the symbol to be either +1 or -1 , MAP or MMSE should be chosen for the early detection process. From Appendix A, we know that with hard decision, $\hat{b}_{p}^{M A P}=\operatorname{sign}\left(x_{p}\right)$ and $\hat{b}_{p}^{M M S E}=\operatorname{sign}\left(\tanh \left(\frac{x_{p}}{\sigma_{x}^{2}}\right)\right)$, which the ARX estimates the symbol value based on the sign of $x_{p}$ for MAP estimator and based on the sign of $\tanh \left(\frac{x_{p}}{\sigma^{2}}\right)$ for MMSE estimator. We assume that the navigation messages have equal probability to be +1 and -1 , we see that MAP and MMSE estimators have the same error probability, based on Eq. 28 and Eq. 34. From the viewpoint of implementing a MAP estimator at the ARX is less complex: because based on derivation of the estimation results, two non-linear function are needed to implement MMSE: tanh and sign, and only one for MAP: sign. As the error probability depends on SNR, the longer the integration time, $T_{E D}$, is, the higher SNR it has, so the better performance the ARX gets.

The early detection performance can also be affected by the interference from other spreading code due to partial spreading code used for the early detection. We know that the Galileo E1 primary codes are optimized pseudo-noise (PN) sequences stored in memory, so called 'memory codes', including E1B and E1C primary codes [14], each set of which consists of 50 members. The PN sequences parameters selected for E1B and E1C are: 1) 4092 chips; 2) $1.023 \times 10^{6} \mathrm{chips} / \mathrm{s}$; 3) same length as a symbol. The partial period correlation occurs when the spreading code sequence is larger than 'one symbol duration', where the symbol duration is $T_{E D}$ at the ARX. The optimized PN sequences are designed in a perfect way with properties to maximize their auto-correlation but minimize their cross-correlation, so that the interference from other PN sequences is very low. The discrete cross-correlation of $P N_{i}$ and $P N_{j}$ is defined by:

$$
C_{i, j}(l)=\left\{\begin{array}{cc}
\sum_{k=0}^{N-1-l} P N_{i}(k+l) P N_{j}(k) & l \geqq 0 \\
C_{i, j}(-l) & l<0
\end{array}\right.
$$

which is normalized by $C_{i, j, \text { normalized }}(l)=\frac{1}{\sqrt{C_{i i}(0) C_{j j}(0)}} C_{i, j}(l)$ [20]. However, these properties become weaker when the length of PN sequences become shorter, as shown in Fig. 2. We can see that the normalized cross-correlation is less than 5\% when the PN sequence length is 4092 , but it is almost $15 \%$ when the sequence length is 200 . Therefore, when the adversary chooses 

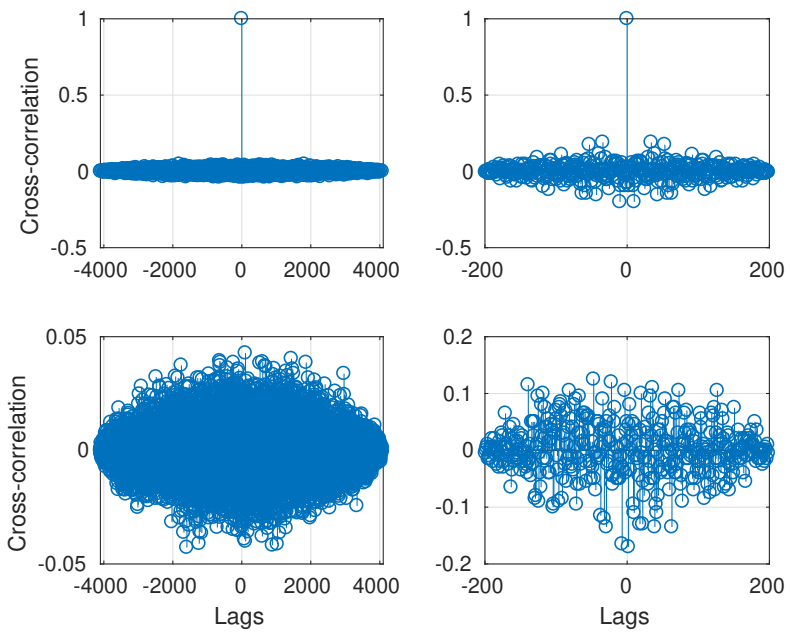

Fig. 2: Normalized auto- and cross-correlation of Galileo E1B PRN5 and PRN25: top-left and top-right are auto-correlation of PRN5 with 4092 chips and 200 chips, separately; bottom-left and bottom-right is cross-correlation of PRN5 and PRN25 with 4092 chips and 200 chips, separately

an early detection time $T_{E D}$, it should consider the effect of the interference from other spreading codes. While considering the interference of other PN codes, the output of the matched filter can be defined as:

$$
\zeta_{i}=\left\{\begin{array}{cc}
\sqrt{P_{i} / 2} T_{E D} \cdot f_{s} b_{i}+\sqrt{P_{j} / 2} T_{E D} \cdot f_{s} b_{j} C_{i, j}(l)+n & \text { case } 1 \\
\sqrt{P_{i} / 2} T_{E D} \cdot f_{s} b_{i}+\int_{0}^{T 1} b_{j}^{*} P N_{j}\left(t-T_{1}\right) P R N_{i}(t) d t+\int_{T_{1}}^{T_{E D}} b_{j} P N_{j}\left(t-T_{1}\right) P R N_{i}(t) d t+n & \text { case } 2
\end{array}\right.
$$

where $n$ is noise, $b_{i}$ and $b_{j}$ are two symbol values of two signals; case 1: for $P N_{j}, b_{j}$ is the same symbol over period $T_{E D}$; case 2: for $P N_{j}$, it goes over two symbols $b_{j}^{*}$ and $b_{j}$ consecutively. For both case 1 and case 2, the cross-correlation variance can be added to the noise. The variance is written for the two cases [17]:

$$
\operatorname{Var}\left(\zeta_{i}\right)=\left\{\begin{array}{cc}
\sqrt{P_{i} / 2} T_{E D} \cdot b_{i}+\sum_{\substack{j=1 \\
j \neq i}}^{J} \sqrt{P_{j} / 2} T_{E D} \cdot b_{j} C_{i, j}(l)+\frac{1}{4} N_{0} T_{E D} & \text { case 1 } \\
\frac{P_{j} T_{E D}^{2} \sum_{\substack{j=1 \\
j \neq i}}^{J}\left[2 \mu_{i, j}(0)+\mu_{i, j}(1)\right]}{12\left(T_{E D} f_{S}\right)^{3}}+\frac{1}{4} N_{0} T_{E D} & \text { case 2 }
\end{array}\right.
$$

where $\mu_{i, j}(0)=\sum_{l=0}^{T_{E D}-1}\left[C_{i, j}^{2}\left(l-T_{E D}+1\right)+C_{i, j}^{2}(l+1)\right]$ and $\mu_{i, j}(1)=\sum_{l=0}^{T_{E D}-1}\left[C_{i, j}\left(l-T_{E D}\right) C_{i, j}\left(l-T_{E D}+1\right)+C_{i, j}(l) C_{i, j}(l+1)\right]$. This leads to a new SNR(signal-to-interference-plus-noise ratio):

$$
S N R=\frac{\sqrt{P_{i} / 2} T_{E D}}{\sqrt{\operatorname{Var}\left(\zeta_{i}\right)}}
$$

We can see that SNR is a function of $T_{E D}$, the effects of this new SNR on BER will be investigated in future work.

\section{Late Commit}

In the late commit (LC) phase, the adversary crafts the DD signals based on the information provided by the ARX. In [3], four different approaches were discussed, on how to craft the transmitted signals for each bit/symbol/chip. The choices that can be applied to the Galileo E1-OS signals are: 1) the pre-defined part for each symbol is noise; 2) the predefined part is the same value as the last symbol.

For the first approach, because the ATX transmits the pre-defined part with noise, the noise power must be very low to avoid introducing significant interference to the accumulation result of each symbol at the HRX. For the second approach, the pre-defined LC part being the last symbol value can be same or opposite to current symbol value $( \pm 1)$. The signal assembled by the ATX is written as:

$$
S_{A T X}(t)= \begin{cases}A_{1} f_{1}(t) & 0 \leq t<T_{L C} \\ A_{2} f_{2}(t) & T_{L C} \leq t \leq T_{b}\end{cases}
$$

where $f_{1}(t)$ is a function of the predefined symbol value, spreading code and carrier, and $f_{2}(t)$ is a function of the ARX value, spreading code and carrier. For both approaches, because the second part of each symbol is assembled based on the 


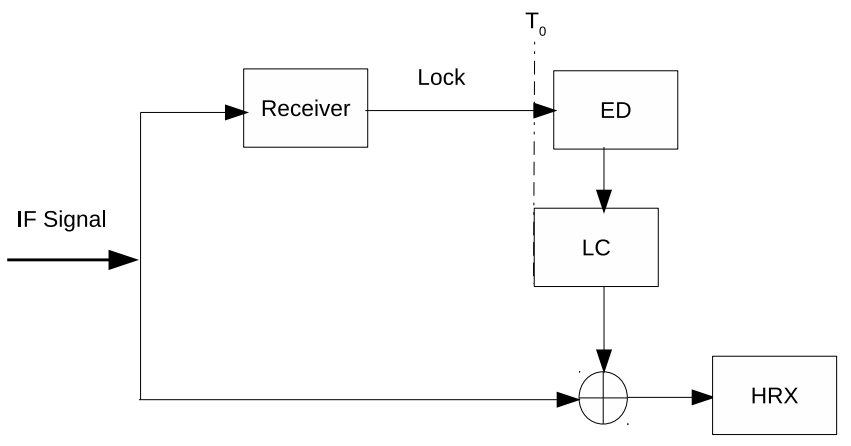

Fig. 3: Synthesized DD attacks signals

ARX value that matches likely the true symbol value, so the accumulated energy should be dominated by the second part. So we have:

$$
A_{2} \cdot\left(T_{b}-T_{L C}\right)>A_{1} \cdot T_{L C}
$$

$A_{1}$ and $A_{2}$ two parameters that can be configured at the ATX, then Eq. 12 is rewritten as:

$$
T_{L C}<\frac{A_{2}}{A_{1}+A_{2}} T_{b}
$$

Based on above equation, we can see that $T_{L C}$ should be smaller than half of the generic Galileo integration period, i.e., $4 \mathrm{~ms}$, when $A_{1}=A_{2}$. However, considering to compensate the processing delay and the early detection time, i.e., $T_{D D}=T_{L C}-T_{E D}-T_{d}$ where the processing delay $T_{d}$ could be in the order of a millisecond and the early detection time could be half millisecond, $T_{L C}$ should be larger than $1.5 \mathrm{~ms}$, otherwise the DD attack would not shorten the distance measurement. Therefore, $A_{1}$ and $A_{2}$ should be configured properly, so that the adversary will have more flexibility to set the early detection time and higher effect on $T_{D D}$.

Recall that at the onset of the DD attack, the adversary basically has two options: 1) it jams the reception of the authentic signals at the HRX to force it lose its tracking, then transmits the DD signals with higher power; 2) the adversary implements a smooth take-over attack, which, however, is difficult in practice. With jamming, the adversary forces the HRX to do reacquisition of available satellite signals. The signal acquisition process at the HRX requires no inner-symbol transition during one integration period that is one spreading code length, i.e., 4 ms. Therefore, the ATX must take this into consideration while crafting each symbol to make sure that the HRX acquires the DD signals instead of the authentic signals. For example, the HRX will not acquire the DD signals if the predefined (LC) part is different from the ARX value, because of the inner-symbol transition. Consequently, the victim will not be locking to the DD signals. Therefore, the attacker can take advantage of the known preamble code of the E1 I/NAV message. The ATX starts assembling the DD signals from the first symbol of the preamble code, in which case the attacker can guarantee that at least the preamble code are transmitted perfectly, because the code is known in prior.

\section{Simulation SETUP}

We first present how we synthesize the DD attacks. Hereafter, we evaluate the receiver performance under our synthesized DD attack. The digitalized RF signal we used for the evaluation is recorded by NT1065_USB3 [21] after the receiver front-end, with four Galileo E1 satellite signals: PRN[3, 5, 9, 22].

The work flow of synthesizing the attack signals is shown in Fig. 3: the ARX tracks and locks on to one signal; the adversary decides to start attacking at receiver time $T_{0}$, the starting edge of one symbol, which is the symbol assembling time at the ATX. After $T_{D D}$, the actual data arrives at the ARX. The transmission time of the DD signal is $T_{D D}$ earlier than reception time of the actual data. The crafted signal is mixed with the original baseband signal that includes all the authentic signals, then the mixed signal is fed to the HRX.

First, we evaluate the error probability of the early detection phase, for different SNR and $T_{E D}$. The SNR range used here is not the value estimated directly from the signal with different integration time $T_{E D}$. In contrast, we add simulated Gaussian noise to the samples during $T_{E D}$ to manually change the SNR of the signals used for early detection for the sake of evaluation, so that we can evaluate the performance based on a large range of SNR. The evaluation process is as follows: we first estimate the carrier-to-noise ratio (CNO) based on the correlator outputs; then we convert the CN0 to true SNR at the receiver with the 


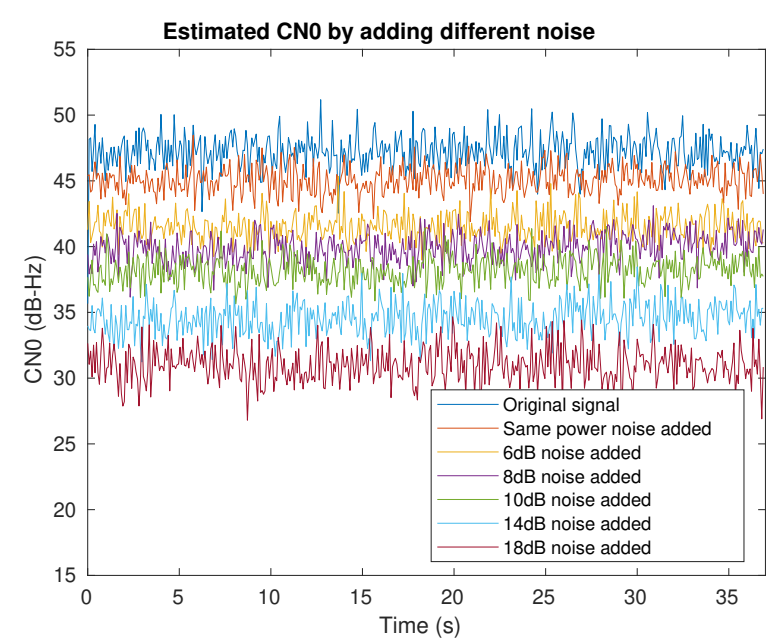

(a) CNO estimation in synthesized environment with added noise for one satellite signal

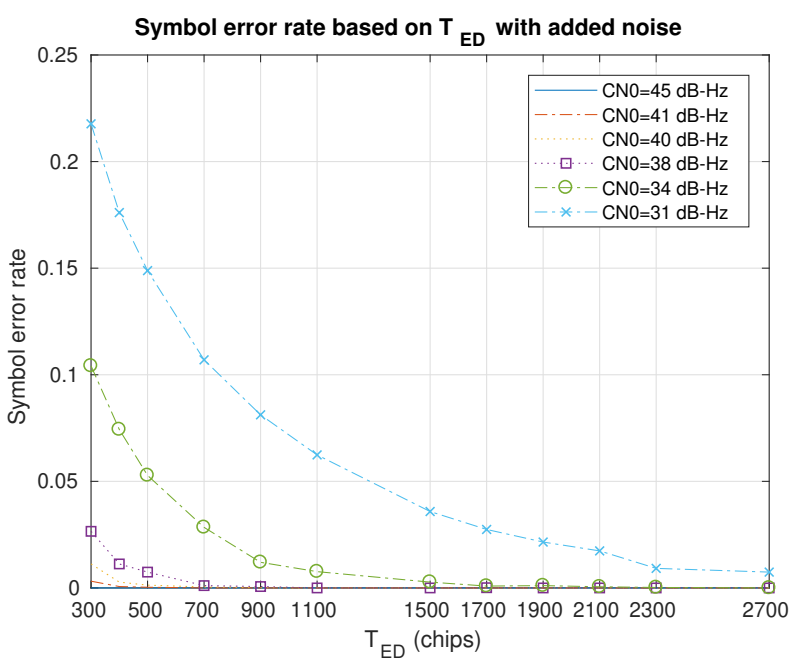

(b) Symbol error rate of the dataset with different synthesized $\mathrm{CNO}$

Fig. 4: Performance evaluation of the ARX

knowledge of the front-end bandwidth; then, generate Gaussian noise with different SNR based on the true SNR; finally, the error rate can be evaluated based on the different SNR and $T_{E D}$. Fig. 4 a shows that the noise environment, CN0 $=31 \sim 45 \mathrm{~dB}-$ $\mathrm{Hz}$, we synthesized in order to evaluate the early detection performance in the ARX, considering that the generally acceptable CNO is $30 \mathrm{~dB}-\mathrm{Hz}$. In the dataset, the estimated original CN0 of the signal is $47 \mathrm{~dB}-\mathrm{Hz}$ (dark blue in Fig. 4a). High CN0 situation is more valuable for evaluation since the ARX is mounted at a clear open high location. We evaluate the early detection performance at the ARX with the synthesized noise environment accordingly, as shown in Fig. 4b. This essentially provides the adversary a "look-up table" to choose a proper $T_{E D}$, based on its environment. In principle, the adversary wants to use as low $T_{E D}$ as possible for early detection, so that $T_{L C}$ and $T_{D D}$ have more options, given $T_{D D}=T_{L C}-T_{E D}$. However, Fig. $4 \mathrm{~b}$ shows that the early detection introduces errors when $T_{E D}<500$ chips when $\mathrm{CN} 0=40 \mathrm{~dB}-\mathrm{Hz}$. When the environment is noisier, $\mathrm{CNO}<40 \mathrm{~dB}-\mathrm{Hz}, T_{E D}$ needs to be very high in order to avoid introducing errors. Moreover, for $\mathrm{CN} 0=31 \mathrm{~dB}-\mathrm{Hz}$, because $T_{E D}$ needs to be large for zero-error detection, thus, $T_{L C}$ needs to be large accordingly; consequently, the ATX needs to transmit a much higher power for the second part of the DD signal, which can be an indication of an attack. By being deployed at a good location, e.g., $\mathrm{CNO}>45 \mathrm{~dB}-\mathrm{Hz}$ in Fig. 4b, the ARX will not introduce symbol errors during the early detection phase even with $T_{E D}$ being a couple of hundred chips length.

The simulated ATX assembles the first several symbols with the preamble code of the signal, allowing the HRX to acquire the DD signals correctly. The preamble code occurs every two seconds for the Galileo E1OS signals, therefore, the ARX has exact information about when next preamble code will come. This information is provided to the ATX, thus, the ATX can start assembling the DD signals $T_{D D}$ ahead of the actual preamble code reception time.

\section{Countermeasure based on GoOdness of Fit TeST}

We propose one detection method for the DD attacks based on a statistical test, which can be implemented in a Galileo receiver with a software patch. The method can provide a real-time solution for identifying DD attacks. The DD attack signal has a special feature that can be used to counter the attack: transition inside each symbol. The reason is that in the late commit phase, the signal for each symbol has two independent parts, a within symbol transition. For legitimate signals, the prompt correlator of the HRX provides energy accumulation over the integration period, $T_{i n t}$ :

$$
\begin{aligned}
I_{P}^{0} & =\sum_{n=1}^{T_{\text {int }} * f_{s}} R(n-\tau) * S_{E 1 B}(n-\tau) \cos \left(2 \pi f_{d} / f_{s} n+\phi\right)+N \\
& \stackrel{\text { filtering }}{=} \frac{\sqrt{P}}{2} \cdot f_{s} \cdot T_{\text {int }} \cdot b+N
\end{aligned}
$$

where $\mathrm{b}$ is the data value: $\{+1,-1\}$. Therefore, with $E=\frac{\sqrt{P}}{2} \cdot f_{s} \cdot T_{i n t}, I_{P}^{0}$ follows:

$$
I_{P}^{0}=b \cdot E+N
$$



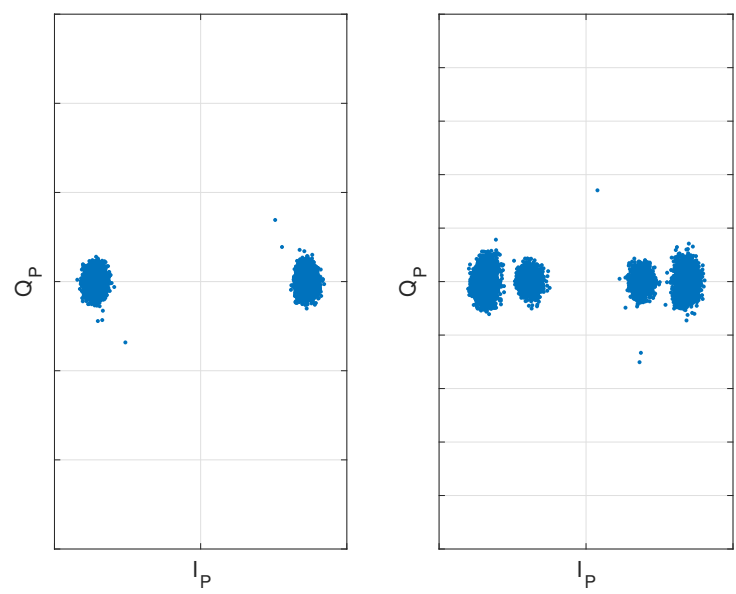

(a) $I_{P}$ and $Q_{P}$ between authentic signal (left) and DD signal (right)

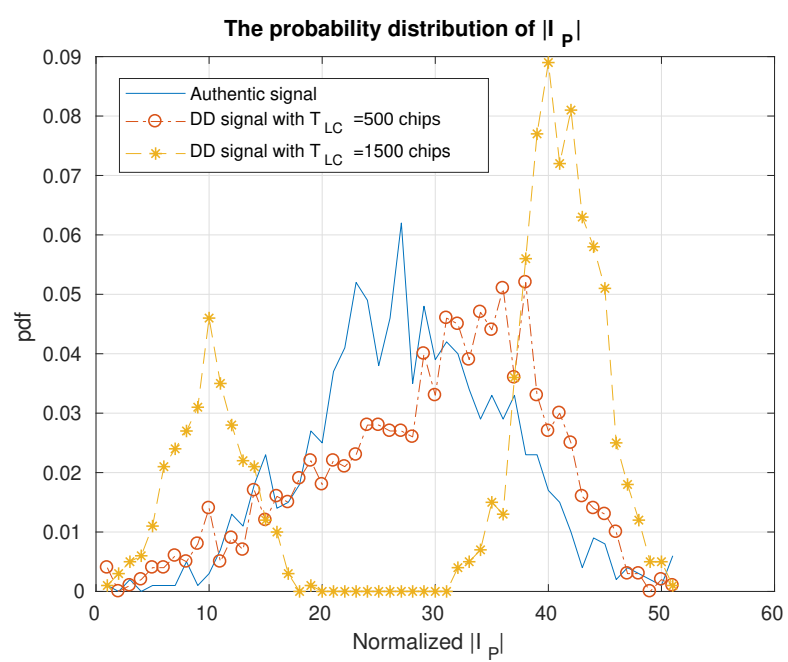

(b) Normalized $\left|I_{P}\right|$ distribution

Fig. 5: Illustration of the difference of prompt correlators

For the DD signals, the prompt correlator provides:

$$
\begin{aligned}
I_{P}^{D D} & =\sum_{n=1}^{T_{L C} * f_{s}} S_{A T X}(n-\tau) * S_{E 1 B}(n-\tau) \cos \left(2 \pi f_{d} / f_{s} n+\phi\right)+\sum_{n=T_{L C} * f_{s}+1}^{T_{\text {inn }} * f_{s}} S_{A T X}(n-\tau) * S_{E 1 B}(n-\tau) \cos \left(2 \pi f_{d} / f_{s} n+\phi\right)+N \\
& =\underline{\text { filtering }} \\
& =b_{\text {pre }} \cdot E \cdot \frac{\sqrt{P}}{2} \cdot f_{s} \cdot T_{L C} \cdot b_{\text {pre }}+A \cdot \frac{\sqrt{P}}{2} \cdot f_{s} \cdot\left(T_{\text {int }}-T_{L C}\right) \cdot b+N \\
T_{\text {int }} & +b \cdot E \cdot \frac{T_{\text {int }}-T_{L C}}{T_{\text {int }}}+N
\end{aligned}
$$

where $b_{\text {pre }}$ is the predefined symbol value. Therefore, $I_{p}^{D D}$ follows:

$$
I_{P}^{D D}=\left\{\begin{array}{cc}
b \cdot E \cdot\left(A+(1-A) \frac{T_{L C}}{T_{\text {int }}}\right)+N & b_{\text {pre }}=b \\
b \cdot E \cdot\left(A-(1+A) \frac{L_{T}}{T_{\text {int }}}\right)+N & b_{\text {pre }}=-b
\end{array}\right.
$$

Assuming a binary signal source, $b_{\text {pre }}$ has $50 \%$ probability to be $b$ or $-b$, therefore, $I_{p}^{D D}$ has equal probability to follow either distribution in Eq. 17. In contrast, $I_{p}^{0}$ follows the distribution in Eq. 15. The visualization of the difference is presented in Fig. 5a: we see clearly the distinction. On the right side of Fig. 5a, the inner pair of 'eyes' are from the DD signals when $b_{p r e}=-b$ and the outer pair of 'eyes' are from the DD signal when $b_{p r e}=b$. Fig. $5 \mathrm{~b}$ provides the normalized distribution of $I_{P}$, which clearly shows that the $I_{P}$ of the DD signals composes of two normal distributions, as presented in Eq. 17. The distance between the two normal distributions, i.e., the difference between two means, is:

$$
\Delta \mu=\left(A+(1-A) \frac{T_{L C}}{T_{\text {int }}}\right)-\left(A-(1+A) \frac{T_{L C}}{T_{\text {int }}}\right)=\frac{2 T_{L C}}{T_{\text {int }}}
$$

which tells us that the resolution between two normal distributions does not depend on the configured amplitude $A$, but only on $T_{L C}$. The bigger $T_{L C}$ is, the more distinguishable the two distributions are. Fig. $5 \mathrm{~b}$ reveals that it is not easy to distinguish the distributions when $T_{L C}=500$ chips. However, as discussed before, $T_{L C}$ should typically be larger than 1500 chips, i.e., $1.5 \mathrm{~ms}$, with a reasonable setting of the early detection time, $T_{E D}=0.5 \mathrm{~ms}$, and processing delay $T_{d}=1 \mathrm{~ms}$, if the adversary aims a gain $T_{D D}>0$, then $T_{L C}=T_{D D}+T_{E D}+T_{d}>1.5 \mathrm{~m}$. In such case, the distinction is easy to find (Fig. $5 \mathrm{~b}$ ).

In order to check which distribution the prompt output fits, $I_{P}^{0}$ or $I_{P}^{D D}$, we design a Anderson-Darling Goodness of Fit (GoF) test to detect DD attacks. The Anderson-Darling test [22] is a statistical test to examine whether observation data samples are drawn from a given probability distribution. When the hypothesis is that the data samples are drawn from a normal distribution, it is one of the most powerful tools to detect the most departures from normality. The Anderson-Darling test, a 


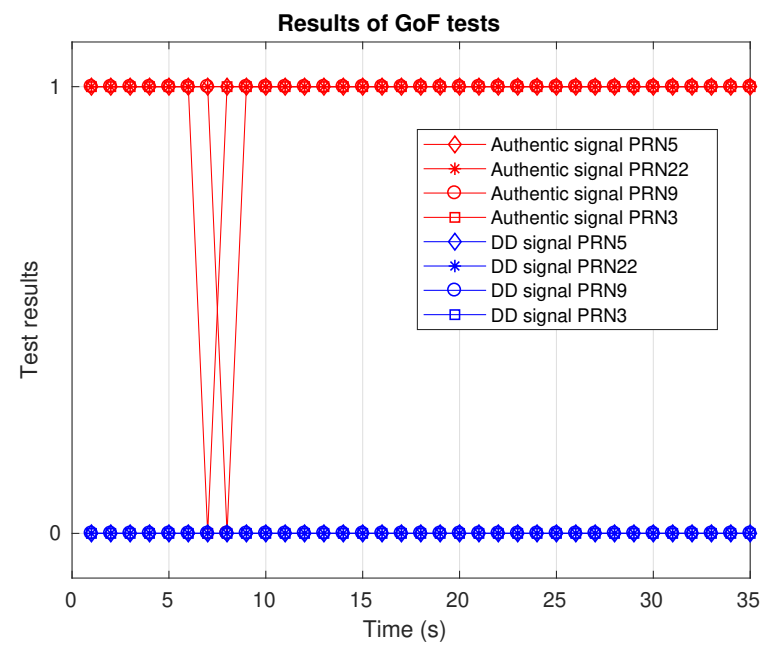

Fig. 6: The Anderson-Darling GoF test results for 35 seconds when $T_{L C}=1500$ chips: "1" means no attacks and "0" implies existence of the DD attacks

quadratic empirical distribution function (EDF) test, measures the distance between the hypothesized cumulative distribution function $(\mathrm{CDF}), F$, and an empirical $\mathrm{CDF}, F_{n}$. The test statistic is written as [23]:

$$
A^{2}=n \int_{-\infty}^{+\infty} \frac{\left(F_{n}(x)-F(x)\right)^{2}}{F(x)(1-F(x))} d F(x)
$$

where $n$ is the number of samples. The statistic is augmented for the case of both unknown mean and variance [24]:

$$
A^{\star 2}=A^{2}\left(1+\frac{0.75}{n}+\frac{2.25}{n^{2}}\right)
$$

As the considered signals are from a binary source, $I_{p}^{0}(n)$ and $I_{p}^{D D}(n)$ are symmetric, therefore, we use the absolute value of the prompt correlator outputs, $\left|I_{p}^{0}(n)\right|$ and $\left|I_{p}^{D D}(n)\right|$, as the observation for the test. Thus, we have:

$$
\left\{\begin{array}{lr}
\text { Authentic hypothesis } & H_{0}: I_{p}(n)=E+N \\
\text { DD attacks hypothesis } & H_{1}: I_{p}(n) \text { does not follows } N\left(E, \sigma^{2}\right)
\end{array}\right.
$$

When $A^{\star 2}$ exceeds a critical value based on a pre-defined significance level, the null hypothesis is rejected; this indicates the detection of the DD attacks. Otherwise, the null hypothesis is accepted, thus implying no DD attack.

In the evaluation, we use a samples size of $n=250$ for each test; the test provides one result per second since each $I_{P}$ is calculated with $4 \mathrm{~ms}$ integration period. We choose a typical significance level 0.01 for the evaluation. Fig. 6 shows test results for both the legitimate/authentic signals and the DD signals: we see that both PRN9 and PRN3 of the legitimate signals have one test rejecting the null hypothesis during 35 seconds. However, for the DD signals, all the tests reject the null hypothesis. In practice, a receiver should raise an alarm for a DD attack with test results being "0" for a consecutive 3-5 tests, i.e., 3-5 seconds, reducing the effect of false positives. The results show that the Anderson-Darling GoF test can detect the DD attacks based on the prompt correlator outputs nearly perfectly, due to its inner-transition of each symbol. Moreover, the detection based on the Anderson-Darling GoF test is powerful in a sense that it can defeat the attacks when the victim is already tracking the adversarial signals. Moreover, the results of the test impose as the adversary modifies the pseudorange measurements with an increased $T_{L C}$.

\section{Conclusions}

The DD attacks can greatly harm the GNSS receivers even in the presence of NMA protection. Specifically, we investigated how the adversary can choose $T_{E D}$ for his best benefit in different noisy environments. The result shows that $T_{E D}$ can be as low as 500 chips to achieve extremely low bit error rate when CN0 is larger than $40 \mathrm{~dB}-\mathrm{Hz}$. Then, we discussed different approaches for the ATX. In practice, the adversary would start transmitting the DD signals from the beginning of a preamble code, in order to be acquired by the HRX during the re-acquisition phase, because the preamble code is known in prior. One special feature of the DD attacks is exploited to propose the statistical test: inner-symbol transition due to that the predefined (LC) part might be opposite to the ARX value. Through the proposed GoF test method, we see that the DD attacks can 
be detected nearly perfectly when $T_{L C}>1500$ chips for the Galileo E1 OS signals. This is also a reasonable value that the adversary would set for $T_{L C}$. The reason is that if the adversary aims a gain $T_{D D}>0$, with a reasonable setting for $T_{E D}=500$ chips, $0.5 \mathrm{~ms}$, and a processing delay $T_{d}=1 \mathrm{~ms}$, then we have $T_{L C}=T_{D D}+T_{E D}+T_{d}>1.5 \mathrm{~ms}$. The proposed method provides a good real time detection when the honest receiver is already tracking the DD signals.

\section{ACKNOWLEDGMENT}

This work has been partially supported by the Swedish Foundation for Strategic Research (SSF) SURPRISE project.

\section{REFERENCES}

[1] T. E. Humphreys, "Detection strategy for cryptographic GNSS anti-spoofing," IEEE Transactions on Aerospace and Electronic Systems, vol. 49, no. 2, pp. 1073-1090, 2013.

[2] K. Zhang and P. Papadimitratos, "GNSS receiver tracking performance analysis under distance-decreasing attacks," in Proceedings of the 2015 International Conference on Location and GNSS (ICL-GNSS 2015), Gothenburg, Sweden, 2015, pp. 1-6.

[3] K. Zhang and P. Papadimitratos, "On the Effects of Distance-decreasing Attacks on Cryptographically Protected GNSS Signals," in Proceedings of the 2019 International Technical Meeting of The Institute of Navigation (ION ITM 2019), Reston, Virginia, 2019, pp. 363-372.

[4] J. A. Volpe, "Vulnerability assessment of the transportation infrastructure relying on the Global PositioningSystem," National Transportation Systems Center, 2001.

[5] I. Fernández-Hernández, V. Rijmen, G. Seco-Granados, J. Simon, I. Rodríguez, and J. D. Calle, "A navigation message authentication proposal for the Galileo open service," NAVIGATION, vol. 63, no. 1, pp. 85-102, 2016.

[6] B. Motella, D. Margaría, and M. Paonni, "SNAP: An authentication concept for the Galileo open service," in Proceedings of the 2018 IEEE/ION Position, Location and Navigation Symposium (PLANS), Monterey, California, 2018, pp. 967-977.

[7] I. Fernández, V. Rijmen, T. Ashur, P. Walker, G. Seco, J. Simón, C. Sarto, D. Burkey, and O. Pozzobon, Galileo Navigation Message Authentication Specification for Signal-In-Space Testing - v1.0. European Commission, 2016.

[8] C. Sarto, O. Pozzobon, S. Fantinato, S. Montagner, I. Hernández, J. Simón, J. D. Calle, S. Cancela, P. Walker, D. Burkey et al., "Implementation and testing of OSNMA for Galileo," in Proceedings of the 2017 International Technical Meeting of The Institute of Navigation (ION GNSS+ 2017), Portland, Oregon, 2017.

[9] A. Perrig, R. Canetti, J. D. Tygar, and D. Song, "The TESLA broadcast authentication protocol," RSA Cryptobytes, vol. 5, no. 2, pp. 2-13, 2002.

[10] R. E. Phelts, D. M. Akos, and P. Enge, "Robust signal quality monitoring and detection of evil waveforms," in Proceedings of the 13th International Technical Meeting of the Satellite Division of the Institute of Navigation, 2000, pp. 1180-1190.

[11] M. Pini, M. Fantino, A. Cavaleri, S. Ugazio, and L. L. Presti, "Signal quality monitoring applied to spoofing detection," in Proceedings of the 24th International Technical Meeting of The Satellite Division of the Institute of Navigation (ION GNSS 2011), 20011, pp. 1888-1896.

[12] M. Pini, B. Motella, and M. T. Gamba, "Detection of correlation distortions through application of statistical methods," in Proceedings of the 26th International Technical Meeting of The Satellite Division of the Institute of Navigation (ION GNSS+ 2013), 2013, pp. 3279-3289.

[13] M. T. Gamba, M. D. Truong, B. Motella, E. Falletti, and T. H. Ta, "Hypothesis testing methods to detect spoofing attacks: A test against the texbat datasets," GPS solutions, vol. 21, no. 2, pp. 577-589, 2017.

[14] The European Union, "Galileo Open Service Signal-In-Space Interface Control Document (OS SIS ICD), Issue 1.2," 2015.

[15] T. E. Humphreys, J. A. Bhatti, D. Shepard, and K. Wesson, "The Texas spoofing test battery: Toward a standard for evaluating GPS signal authentication techniques," in Radionavigation Laboratory Conference Proceedings, 2012.

[16] T. E. Humphreys, B. M. Ledvina, M. L. Psiaki, B. W. O’Hanlon, and P. M. Kintner, "Assessing the spoofing threat: Development of a portable GPS civilian spoofer," in Radionavigation laboratory conference proceedings, 2008.

[17] M. K. Simon, J. K. Omura, R. A. Scholtz, and B. K. Levitt, Spread Spectrum Communications Handbook. Citeseer, 1994.

[18] M. H. Zarrabizadeh and E. S. Sousa, "A differentially coherent pn code acquisition receiver for cdma systems," IEEE Transactions on communications, vol. 45, no. 11, pp. 1456-1465, 1997.

[19] G. P. John and S. Masoud, Digital Communications 5th Edition. McGrad-Hill, 2007.

[20] P. Stoica, R. L. Moses et al., Spectral Analysis of Signals. Pearson Prentice Hall Upper Saddle River, NJ, 2005.

[21] “NT1065_USB3 Module," http://ntlab.com/section/sec:v:44979.htm, accessed: 2019-06-19.

[22] T. W. Anderson, D. A. Darling et al., "Asymptotic theory of certain" goodness of fit" criteria based on stochastic processes," The annals of mathematical statistics, vol. 23, no. 2, pp. 193-212, 1952.

[23] T. W. Anderson and D. A. Darling, "A test of goodness of fit," Journal of the American statistical association, vol. 49, no. 268 , pp. 765-769, 1954.

[24] R. B. D’Agostino, “Tests for the normal distribution,” in Goodness-of-fit-techniques. Routledge, 2017 , pp. 367-420.

[25] T. Kailath, A. H. Sayed, and B. Hassibi, Linear Estimation. Prentice Hall, 2000.

\section{APPENDiX A \\ Three Estimation Algorithms}

\section{A. Maximum Likelihood (ML) Estimator}

The ML estimation is to obtain $\hat{b_{p}}$ of Eq. 6 as follows:

$$
\hat{b}_{p}^{M L}=\max _{b_{p}} p\left(x_{p} \mid b_{p}\right)
$$

which gives the symbol estimate that makes the observation $x_{p}$ most likely. Because the ARX attempts to estimate the symbol value at end of the $T_{E D}$ length fraction of each symbol, so there is only one observation of $x_{p}$ for each symbol. Therefore, the symbol value that makes the observation $x_{p}$ most likely is the observation itself:

$$
\hat{b}_{p}^{M L}=x_{p}
$$




\section{B. Maximum A Posteriori (MAP) Estimator}

The ML estimation does not consider the prior distribution of the symbol value $b_{p}$, unlikely, the MAP estimator:

$$
\hat{b}_{p}^{M A P}=\max _{b_{p}} p\left(b_{p} \mid x_{p}\right)=\max _{b_{p}} \frac{p\left(x_{p} \mid b_{p}\right) p\left(b_{p}\right)}{p\left(x_{p}\right)}=\max _{b_{p}} p\left(x_{p} \mid b_{p}\right) p\left(b_{p}\right)
$$

We have prior information on the symbol value, $b_{p}$. It can be +1 or -1 , thus, Eq. 6 is rewritten as:

$$
x_{p}= \begin{cases}+E+n \sim N\left(+E, \sigma_{x}^{2}\right) & b_{p}=+1 \\ -E+n \sim N\left(-E, \sigma_{x}^{2}\right) & b_{p}=-1\end{cases}
$$

Therefore, the MAP estimation can be rewritten as:

$$
\begin{gathered}
\hat{b}_{p}^{M A P}=+1 \\
p\left(b_{p}=+1\right) p\left(x_{p} \mid b_{p}=+1\right) \underset{b_{p}^{M A P}}{\gtrless} p\left(b_{p}=-1\right) p\left(x_{p} \mid b_{p}=-1\right) \Longrightarrow \\
\hat{b}_{p}^{M A P}=+1 \\
p\left(b_{p}=+1\right) p_{N\left(+E, \sigma_{x}^{2}\right)}\left(x_{p}\right) \underset{b_{p}^{M A P}}{\gtrless} p\left(b_{p}=-1\right) p_{N\left(-E, \sigma_{x}^{2}\right)}\left(x_{p}\right)
\end{gathered}
$$

This is a hard decision on the symbol value based on the observation $x_{p}$.

As this is a binary antipodal signal, i.e., $b_{p}=\{+1,-1\}$ with probabilities of $p$ and $1-p$, respectively, the error probability is [19]:

$$
P_{e}=p Q\left(\frac{\sqrt{E_{T_{E D}}}-r_{t h}}{\sigma_{x}}\right)+(1-p) Q\left(\frac{\sqrt{E_{T_{E D}}}+r_{t h}}{\sigma_{x}}\right)
$$

where $E_{T_{E D}}$ is the energy of period $T_{E D}$ and $r_{t h}$ is the threshold used to decide whether $b_{p}=+1$ or $b_{p}=-1$.

For a special case, i.e., $p=0.5$, which implies $r_{t h}=0$, the error probability is:

$$
P_{e}=Q\left(\sqrt{\frac{E_{T_{E D}}}{\sigma_{x}^{2}}}\right)
$$

\section{Minimum Mean Square Error (MMSE) Estimator}

An MMSE estimator minimizes the mean square error given an observation data:

$$
\hat{b}_{p}^{M M S E}=\min _{\hat{b_{p}}} E\left\{\left(b_{p}-\hat{b_{p}}\right)^{2} \mid x_{p}\right\}
$$

When the mean and variance are finite, the MMSE estimator is [25]:

$$
\hat{b}_{p}^{M M S E}=E\left\{b_{p} \mid x_{p}\right\}
$$

which is the conditional expectation of $b_{p}$ given $x_{p}$. Thus:

$$
\begin{aligned}
& \hat{b}_{p}^{M M S E}=E\left\{b_{p} \mid x_{p}\right\} \stackrel{b_{p}= \pm 1}{=}(+1) \times p\left(+1 \mid x_{p}\right)+(-1) \times p\left(-1 \mid x_{p}\right) \\
& \underline{\underline{p\left\{b_{p}=+1\right\}+p\left\{b_{p}=-1\right\}=1}} 2 p\left(b_{p}=+1 \mid x_{p}\right)-1
\end{aligned}
$$

With the posterior probability $p\left(b_{p}=+1 \mid x_{p}\right)$ derived in Appendix B, we have:

$$
\hat{b}_{p}^{M M S E}=\frac{2 p\left(b_{p}=+1\right)}{p\left(b_{p}=+1\right)+\left(1-p\left(b_{p}=+1\right)\right) \exp \left(-\frac{2 x_{p}}{\sigma_{x}^{2}}\right)}-1
$$

The posterior probability distribution can be obtained when the prior probability distribution of $p\left(b_{p}\right)$ is known. If the symbol source generates \pm 1 with equal probability, i.e., $p\left(b_{p}=+1\right)=p\left(b_{p}=-1\right)=\frac{1}{2}$, then the posterior probability is:

$$
p\left(b_{p}=+1 \mid x_{p}\right)=\frac{1}{1+\exp \left(-\frac{2 x_{p}}{\sigma_{x}^{2}}\right)}
$$

Based on Eq. 32, given the symmetric binary navigation symbols, we have the MMSE estimation value of the symbol: $\hat{b}_{p}^{M M S E}=\left(1-\exp \left(-\frac{2 x_{p}}{\sigma_{x}^{2}}\right)\right) /\left(1+\exp \left(-\frac{2 x_{p}}{\sigma_{x}^{2}}\right)\right)=\tanh \left(\frac{x_{p}}{\sigma_{x}^{2}}\right)$. The goal of the ARX is to determine the symbol value being +1 or 
-1 , given $\hat{b}_{p}^{M M S E}$ that is bounded $-1<\hat{b}_{p}^{M M S E}<1$. As the symbol is generated by a binary symmetric source, the decision can be made by the ARX: the symbol is +1 if $\hat{b}_{p}^{M M S E}>0$ and the symbol is -1 if $\hat{b}_{p}^{M M S E}<0$.

With equiprobable messages, the error probability for the above decision is:

$$
\begin{aligned}
P_{e} & =p\left(b_{p}=+1\right) p\left(\hat{b}_{p}^{M M S E}<0 \mid b_{p}=+1\right)+p\left(b_{p}=-1\right) p\left(\hat{b}_{p}^{M M S E}>0 \mid b_{p}=-1\right) \\
& =\frac{1}{2} p\left(\tanh \left(\frac{x_{p}}{\sigma_{x}^{2}}\right)<0 \mid b_{p}=+1\right)+\frac{1}{2} p\left(\tanh \left(\frac{x_{p}}{\sigma_{x}^{2}}\right)>0 \mid b_{p}=-1\right) \\
& =\frac{1}{2} p\left(\tanh \left(\frac{+E+N(n)}{\sigma_{x}^{2}}\right)<0\right)+\frac{1}{2} p\left(\tanh \left(\frac{-E+N(n)}{\sigma_{x}^{2}}\right)>0\right) \\
& =\frac{1}{2} p\left(\frac{+E+N(n)}{\sigma_{x}^{2}}<0\right)+\frac{1}{2} p\left(\frac{-E+N(n)}{\sigma_{x}^{2}}>0\right) \\
& =\frac{1}{2} p((+E+N(n))<0)+\frac{1}{2} p((-E+N(n))>0) \\
& =Q\left(\sqrt{\frac{E}{\sigma_{x}^{2}}}\right)
\end{aligned}
$$

where $E$ is the energy of period of $T_{E D}$, i.e., $E_{T_{E D}}$. The error probability turns out to be equal to the error probability of the MAP estimation.

\section{ApPendix B \\ Derives of Posterior Distribution of Symbol Value}

Given the linear observation model in Eq. 6, the posterior probability distribution of the symbol value given one observation is calculated based on the Bayes theorem:

$$
p_{B_{p} \mid X=x_{p}}\left(b_{p}\right)=\frac{p_{B_{p}}\left(b_{p}\right) p\left(x_{p} \mid b_{p}\right)}{\int_{-\infty}^{+\infty} p_{B_{p}}(u) p_{X \mid B_{p}=u}\left(x_{p}\right) d u}
$$

We have the prior information of the symbol value $B=\{+1,-1\}$, thus, Eq. 35 is rewritten as:

$$
p_{B_{p} \mid X=x_{p}}\left(b_{p}\right)=\frac{p_{B_{p}}\left(b_{p}\right) p\left(x_{p} \mid b_{p}\right)}{p\left(b_{p}=+1\right) p_{X \mid b_{p}=+1}\left(x_{p}\right)+p\left(b_{p}=-1\right) p_{X \mid b_{p}=-1}\left(x_{p}\right)}
$$

The posterior probability distribution only has two values: $p_{B_{p} \mid X=x_{p}}\left(b_{p}=+1\right)$ and $p_{B_{p} \mid X=x_{p}}\left(b_{p}=-1\right)$ and $p_{B_{p} \mid X=x_{p}}\left(b_{p}=\right.$ $+1)+p_{B_{p} \mid X=x_{p}}\left(b_{p}=-1\right)=1$. Given $p\left(b_{p}=+1\right)+p\left(b_{p}=-1\right)=1$, together with Eq. 6 , we can get the posterior probability for $b_{p}=+1$ :

$$
\begin{aligned}
p\left(b_{p}=+1 \mid x_{p}\right) & =\frac{p\left(b_{p}=+1\right) p\left(x_{p} \mid b_{p}=+1\right)}{p\left(b_{p}=+1\right) p\left(x_{p} \mid b_{p}=+1\right)+p\left(b_{p}=-1\right) p\left(x_{p} \mid b_{p}=-1\right)} \\
& =\frac{p\left(b_{p}=+1\right) \frac{1}{\sqrt{2 \pi \sigma_{x}^{2}}} \exp \left(-\frac{\left(x_{p}-1\right)^{2}}{2 \sigma_{x}^{2}}\right)}{p\left(b_{p}=+1\right) \frac{1}{\sqrt{2 \pi \sigma_{x}^{2}}} \exp \left(-\frac{\left(x_{p}-1\right)^{2}}{2 \sigma_{x}^{2}}\right)+\left(1-p\left(b_{p}=+1\right)\right) \frac{1}{\sqrt{2 \pi \sigma_{x}^{2}}} \exp \left(-\frac{\left(x_{p}-1\right)^{2}}{2 \sigma_{x}^{2}}\right)} \\
& =\frac{p\left(b_{p}=+1\right)}{p\left(b_{p}=+1\right)+\left(1-p\left(b_{p}=+1\right)\right) \exp \left(-\frac{2 x_{p}}{\sigma_{x}^{2}}\right)}
\end{aligned}
$$

